\title{
O ponto de equilíbrio na assimilação de carbono em sistemas agroflorestais nos municípios de Cametá e Tomé-Açu, no estado do Pará, Brasil \\ The balance point on carbon assimilation in agroforestry systems in the municipalities of Cametá and Tomé-Açu, State of Pará, Brazil
}

\author{
Silvio Roberto Miranda dos Santos', Osvaldo Ryohei Kato", Manoel Malheiros Tourinho', \\ José Felipe Souza de Almeidal, Beatriz Lopes Pereira \\ Universidade Federal Rural da Amazônia. Belém, Pará, Brasil \\ "Empresa Brasileira de Pesquisa Agropecuária. Belém, Pará, Brasil
}

\begin{abstract}
Resumo: $\bigcirc$ estudo objetivou avaliar o ponto de equilíbrio entre fatores bióticos e abióticos na assimilação de carbono em sistemas agroflorestais (SAF), nos municípios de Cametá (SAF-CM) e de Tomé-Açu (SAF-TA), no estado do Pará. Utilizou-se oito parcelas de $50 \mathrm{~m} \times 50 \mathrm{~m}$, onde foram medidos o diâmetro à altura do peito (DAP) e a altura (H) de todos os indivíduos $\left(n_{i}\right) c o m$ DAP $\geq 5 \mathrm{~cm}$. Para determinar o estoque de carbono (EC) contido na biomassa seca, a vegetação dos SAF foi dividida em açaí (Euterpe oleracea), cacau (Theobroma cacao) e árvores (espécies florestais). Foram inventariados, em média, 2.458 n:/ha nos SAF-CM e 1.249 n:/ha nos SAF-TA, sendo açaí e cacau as espécies mais importantes. O EC foi maior nas árvores tanto nos SAF-CM quanto nos SAF-TA. A modelagem matemática presa-predador nas populações de carbono e de plantas nos SAF demonstrou elevado número de ponto de equilíbrio, que, na prática, seria o melhor momento para conduzir um plano de manejo. $O$ modelo mostrou-se apto e adequado na avaliação da interação e do ponto de equilíbrio entre as populações (carbono e plantas). Os resultados ratificam os SAF como uma alternativa de produção sustentável do ponto de vista ambiental e socioeconômico para a região e para a Amazônia.
\end{abstract}

Palavras-chave: Relações sistêmicas. Modelo presa-predador. Biomassa.

\begin{abstract}
The objective of this study was to evaluate the equilibrium between biotic and abiotic factors in the assimilation of carbon in agroforestry systems (SAF), in the municipalities of Cametá (SAF-CM) and of Tomé-Açu (SAF-TA), State of Pará. Eight plots of $50 \mathrm{~m} \times 50 \mathrm{~m}$ were used, where the diameter at breast height $(\mathrm{DBH})$ and height $(\mathrm{H})$ of all individuals $\left(\mathrm{n}_{\mathrm{i}}\right)$ with $\mathrm{DBH}$ $\geq 5 \mathrm{~cm}$ were measured. To determine the Carbon Stock (EC) contained in the dry biomass, the vegetation of the SAF was divided into: açaí (Euterpe oleracea), cacao (Theobroma cacao) and trees (forest species). In an average of 2,458 n/ha in SAF-CM and 1,249 n:/ha in SAF-TA, açaí and cacao were the most important species. In both SAF-CM and SAF-TA, the EC was higher in the trees. Prey-predator mathematical modeling in carbon and plant populations in SAF demonstrated a high number of break-even points, which in practice would be the best time to conduct a management plan. The model proved to be apt and adequate in the evaluation of the interaction and equilibrium point between the populations (carbon and plants). The results confirm the SAF as an alternative of sustainable production from the environmental and socioeconomic point of view for the region and the Amazon.
\end{abstract}

Keywords: Systemic relations. Prey-predator model. Biomass.

SANTOS, S. R. M., O. R. KATO, M. M. TOURINHO, J. F. S. ALMEIDA \& B. L. PEREIRA, 2019. O ponto de equilíbrio na assimilação de carbono em sistemas agroflorestais nos municípios de Cametá e Tomé-Açu, no estado do Pará, Brasil. Boletim do Museu Paraense Emílio Goeldi. Ciências Naturais 14(1): 43-54.

Autor para correspondência: Silvio Roberto Miranda dos Santos. Universidade Federal Rural da Amazônia. Instituo Socioambiental e dos Recursos Hídricos (ISARH). Projeto Várzea. Avenida Presidente Tancredo Neves, 2501 - Terra Firme. Belém, PA, Brasil. CEP 66077-830 (silvio.santos@ufra.edu.br, silviormsantos@gmail.com).

Recebido em 08/06/2017

Aprovado em 27/09/2018

Responsabilidade editorial: Fernando da Silva Carvalho Filho
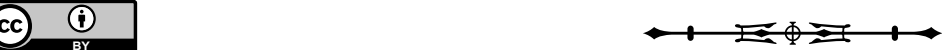


\section{INTRODUÇÃO}

Nos últimos anos, evidenciou-se a mudança climática global e seus efeitos, causados por diversas atividades antrópicas, o que foi ratificado pelo aumento e pela frequência de fenômenos naturais, como secas, tempestades, degelo nos polos etc. (Santos et al., 2004). Diante disso, Sougata \& Shibu (2012) afirmam ser urgente a mudança nos atuais paradigmas de consumo e a definição de uma nova matriz de desenvolvimento, comprometida em minorar perdas ambientais e socioeconômicos decorrentes do uso inadequado dos recursos naturais.

Perante às discussões em torno da necessidade de adequação das atividades produtivas aos conceitos e princípios de sustentabilidade, os sistemas agroflorestais (SAF) têm sido considerados uma das alternativas de uso do solo e de recuperação ambiental (Fassbender, 1993), exatamente porque favorecem a resiliência e a estabilidade (Santos et al., 2004; Porro et al., 2012), além de apresentarem o maior ativo em biomassa (Osterrooht, 2002), colocando-se como sistemas aptos a contribuírem para a produção, a assimilação e o estoque de carbono, reduzindo a ação deste elemento na forma de gás carbônico $\left(\mathrm{CO}_{2}\right)$, um dos principais gases de efeito estufa (GEE) presentes na atmosfera (Dias et al., 2015; Villa et al., 2015).

Na região amazônica, devido à dinâmica da sucessão vegetal e à diversidade natural, a classificação dos SAF pode ser muito variada. Smith et al. (1998) os classificaram como SAF-tradicional - cultivado por comunidades tradicionais e SAF-comercial - cultivado com planejamento e praticado sob orientação e assistência técnica.

A biomassa vegetal de um ecossistema, seja natural e/ou antrópico, é um importante indicador para monitorar a exportação de nutrientes da vegetação e do solo, bem como para nortear iniciativas que visam minimizar impactos ambientais de atividades antrópicas com efeitos potencializadores para a produção de GEE (Higuchi et al., 1998; Ketterings et al., 2001). A biomassa pode ser determinada pelos métodos: a) direto (destrutivo), mais acurado e eficaz, onde geralmente são usadas poucas e pequenas parcelas, e b) indireto (não destrutivo), mais rápido e extensivo, pois utiliza variáveis como o diâmetro e a altura (Higuchi et al., 1998; Hairiah et al., 2001; Dallagnol et al., 2011).

Com o escopo de orientar iniciativas que busquem minimizar impactos ambientais, a teoria geral dos sistemas (TGS) pode ser uma ferramenta relevante a ser mobilizada, pois coloca à disposição da comunidade científica elementos, processos e padrões encontrados na biologia. Entretanto, a TGS não visa resolver problemas e dar soluções imediatas, mas produzir modelos e experiências que possam criar condições aplicáveis à realidade (Bertalanffy, 1968). A TGS conduz à noção de natureza absoluta: biótipo (geográfica ou território) + biocenose (interações entre seres vivos). Tais interações podem ser alteradas pela antropia em ambas as dimensões do ecossistema (Pena-Vega, 2003). Daí a importância das equações diferenciais simultâneas (EDS) para aferição de valores interativos, como nas relações de troca nas quais há incessante conexão entre a parte e o todo (Bertalanffy, 1975).

Dessa forma, o modelo presa-predador de LotkaVolterra, criado independentemente por Lotka em 1925 e por Volterra em 1926 (Lütz, 2011), é de grande importância para os estudos da modelagem matemática de sistemas ecológicos. Trata-se de uma EDS não linear de primeira ordem, comumente usada para descrever dinâmicas nos sistemas biológicos, como preconiza a TGS (Bertalanffy, 1975), quando duas espécies interagem: uma como presa e outra como predadora, que coexistem com a competição, mesmo explorando uma a outra para sobreviverem (Odum et al., 1987; Lütz, 2011).

Alguns sistemas criam e apresentam repetidas oscilações. A qualquer momento, as quantidades aumentam ou diminuem. Um exemplo simples desse sistema é o modelo presa-predador de Lotka-Volterra (Odum et al., 1987). Quando essas quantidades são iguais em tamanho absoluto ou são igualmente proporcionais, não oscilam, gerando a estabilidade física ou numérica, então ocorre o ponto de equilíbrio. A partir desse ponto, é possível observar 
e diferenciar a evolução das populações (presa e predadora). Em um sistema oscilante: quando cresce a presa também cresce a predadora e quando decresce a presa também decresce a predadora (Chase et al., 2014). O modelo estabelece que o número de indivíduos de cada espécie evolui com o passar do tempo e chega ao seu nível 'ótimo' - o qual se traduz graficamente no ponto de equilíbrio, de acordo com o sistema simultâneo e diferencial (Lütz, 2011). Então, o modelo Lotka-Volterra é uma consequência da lei de equilíbrio. Se definirmos $\mathrm{N}(t)$ como o número (ou densidade) de presas e $\mathrm{P}(t)$ como o número (ou densidade) de predadores, o modelo apresenta uma situação em que existem duas espécies que convivem - uma é presa e outra é predadora -, representada pelo conjunto de equações 1 e 2:

$$
\frac{d x}{d t}=a x-b x y
$$

$$
\frac{d y}{d t}=-c y+d x y
$$

Nas equações, $x$ é a população presa e $y$ é a população predadora; a é a taxa de crescimento (ou de natalidade) da população presa; $b$ é a taxa de decrescimento (ou de mortalidade) da população presa; c é a taxa de crescimento (ou de natalidade) da população predadora e $d$ é a taxa de decrescimento (ou de mortalidade) da população predadora. $\mathrm{Na}$ equação 1 (presa), o termo ax indica que as presas crescerão de modo exponencial na ausência de predadores e o termo - bxy implica a redução das presas por ação dos predadores. $\mathrm{Na}$ equação 2 (predador), o termo $-c y$ indica que a população de predadores decai exponencialmente na ausência de presas, e o termo $d x y$ indica que a perda de presas leva à produção de novos predadores (Lütz, 2011).

No contexto ecológico e dos ecossistemas, o modelo Lotka-Volterra, apesar de ser simples, retrata a dinâmica de presas e de predadores, e capta a existência de oscilações ou de periodicidade. Nesse modelo, a dinâmica aumenta e diminui, descrevendo uma trajetória ou órbita fechada, onde as populações evoluem, percorrendo a curva no espaço e no tempo, retornando à posição inicial - o sistema é, portanto, periódico (Odum et al., 1987; Chase et al., 2014). Neste estudo, o modelo foi utilizado com pioneirismo e vanguarda metodológica para analisar as interações entre organismo vivo (planta) e elemento químico (carbono), resultantes tanto de atividades naturais (fotossíntese) quanto antrópicas (desmatamento, agricultura, queima de combustíveis fósseis). Assim, o modelo foi adaptado para esta pesquisa: a presa é o carbono e o predador é a planta (cultivada em SAF).

Considerando-se que os SAF podem desempenhar importante papel, por meio da redução de agravos ambientais e utilizando-se o modelo presa-predador de Lotka-Volterra, para a avaliação da evolução de populações, este trabalho teve como objetivos: a) determinar o estoque de carbono (EC) e b) avaliar o ponto de equilíbrio (PE) na assimilação de carbono nos SAF, implantados nos municípios de Cametá e Tomé-Açu, no estado do Pará.

\section{MATERIAL E MÉTODOS}

O estudo foi realizado nos municípios de Cametá e ToméAçu, ambos pertencentes à mesorregião do nordeste paraense (Figura 1). Cametá está a $184 \mathrm{~km}$ de Belém, ocupa uma área de quase $3.122,9 \mathrm{~km}^{2}$ e apresenta as seguintes coordenadas geográficas: $02^{\circ} 14^{\prime} 40^{\prime \prime} \mathrm{S}$ e $49^{\circ} 29^{\prime}$ 45" W. O clima é quente e úmido, do tipo Ami, conforme a classificação de Köppen. A temperatura média anual é de $26,5^{\circ} \mathrm{C}$, com mínima de $22^{\circ} \mathrm{C}$ e máxima de $31^{\circ} \mathrm{C}$. A umidade relativa do ar é de $85 \%$. A pluviosidade média anual é de $2.375 \mathrm{~mm}$, maior entre janeiro a maio e menor entre junho a dezembro (IBGE, 2012). Tomé-Açu, por sua vez, está a $208 \mathrm{~km}$ de Belém, ocupa uma área de quase $5.145,4 \mathrm{~km}^{2}$, com as seguintes coordenadas geográicas: $02^{\circ}$ 40' 54" S e 48 16' 11' W. O clima é quente e úmido, do tipo Ami, conforme a classificação de Köppen. A temperatura média anual é de $26,8^{\circ} \mathrm{C}$, com mínima de $21^{\circ} \mathrm{C}$ e máxima de $33^{\circ} \mathrm{C}$. A umidade relativa do ar é de $85 \%$. A pluviosidade média anual é de $2.362 \mathrm{~mm}$, maior entre dezembro a maio e menor entre junho a novembro (IBGE, 2012).

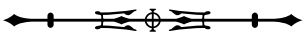




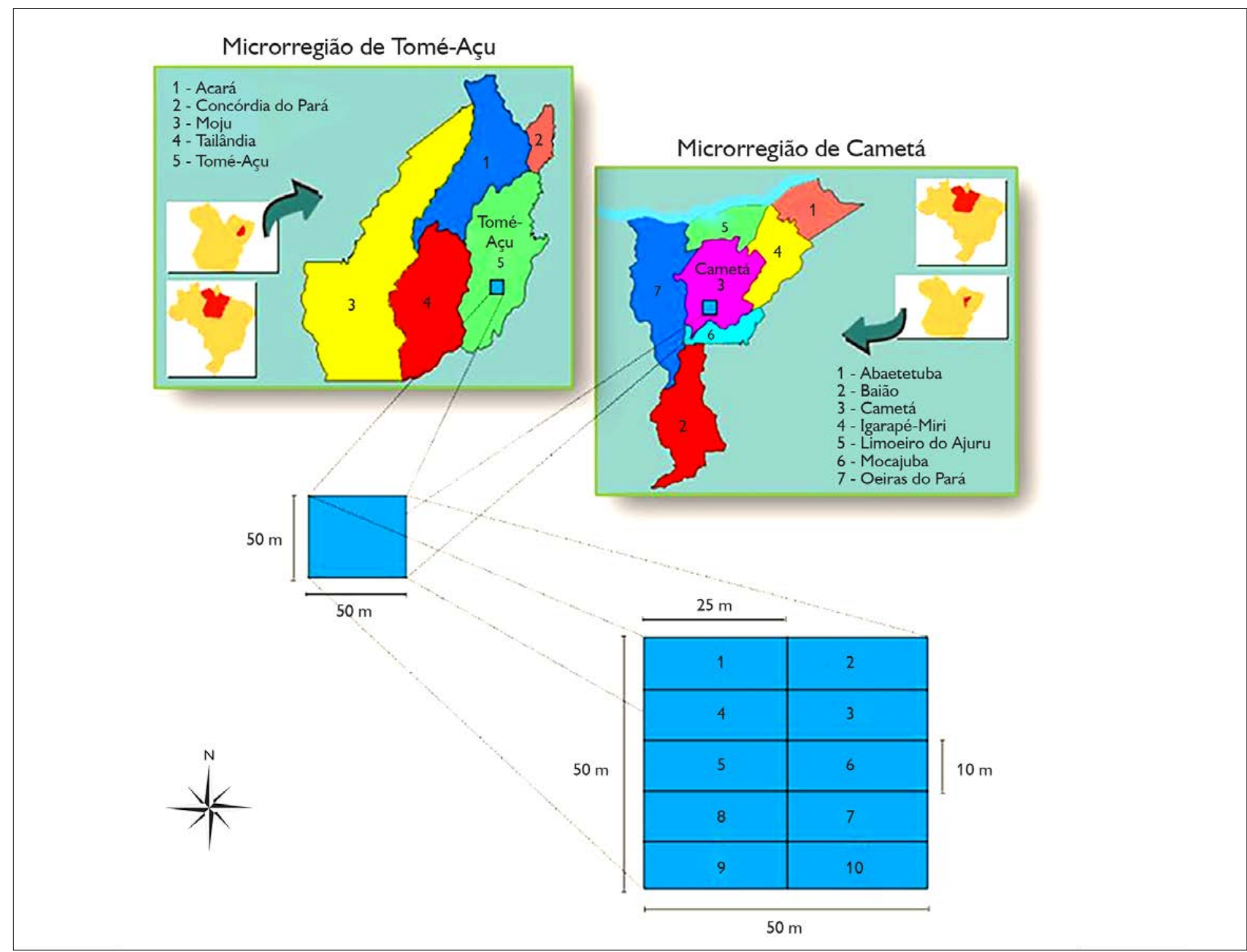

Figura 1. Áreas dos estudos nos municípios de Cametá e de Tomé-Açu, no estado do Pará. Fonte: Santos et al. (2019, p. 33).

O SAF-tradicional é o que melhor caracteriza o que verificamos em Cametá, originado a partir do manejo da floresta natural já explorada, que apresenta cobertura vegetal em torno de $50 \%$ da área e sub-bosque relativamente aberto, com muitas palmeiras e arbustos, enriquecido com espécies frutíferas (principalmente Euterpe oleracea Mart. e Theobroma cacao L.). Esses SAF são submetidos a manejo moderado e esporádico, que visa minimizar impactos no meio ambiente e na área de produção, consistindo em desbastes de açaizeiros de indivíduos mais velhos cuja produção de frutos está reduzindo (aproveitados na produção de palmitos), além de corte de cipós e de espécies invasoras indesejáveis, coletas de sementes, frutos, óleos, cascas e ervas para fins medicinais e uma eventual extração de madeira para uso na propriedade (Santos et al., 2004).

O SAF-comercial, por sua vez, é o que melhor caracteriza o que observamos em Tomé-Açu, sendo o sistema de uso da terra mais comum entre os agricultores nipo-brasileiros da região, uma referência de eficiência técnica, produtividade e viabilidade socioeconômica. Ele começou a ser cultivado em substituição ao monocultivo de pimenta-do-reino (Piper nigrum L.), após o colapso na produção dos pimentais, praticamente dizimados pela fusariose, doença causada pelo fungo Fusarium solani f. sp. piperis, que surgiu na década de 1950 e devastou a região, com ápice na década de 1970 (Homma, 2006).

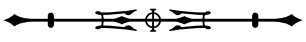


Os agricultores destacam o apoio da Cooperativa Agrícola Mista de Tomé-Açu (CAMTA) na implantação e na condução dos SAF, os quais são submetidos a manejo intensivo, que visa condicionar a área para o incremento das espécies mais importantes para comercialização (Barros, 2009). Esses SAF apresentam algumas particularidades na sua implantação, como a sequência de plantio: pimentado-reino, maracujá (Passiflora edulis Sims), cultura de ciclo curto, frutíferas arbustivas, frutíferas palmeiras, frutíferas arbóreas e espécies florestais (Homma, 2006).

Foram selecionadas oito propriedades com cultivo em SAF com diferentes idades, sendo quatro localizdas em Cametá - com dez, 11, 13 e 14 anos, respectivamente, SAF.1-CM, SAF.2-CM, SAF.3-CM e SAF.4-CM- e quatro em Tomé-Açu - com três, nove, 15 e 21 anos, respectivamente, SAF.1-TA, SAF.2-TA, SAF.3-TA e SAF.4-TA. A média de idade desses SAF é de 12 anos. Nessas unidades, foram inventariadas três parcelas amostrais de $50 \mathrm{~m} \times 50 \mathrm{~m}$, em um total de doze parcelas, divididas em dez subparcelas de $10 \mathrm{~m}$ $\times 25$ m (Figura 1), onde foram medidos, com fita diamétrica, o diâmetro à altura do peito (DAP) e, com vara de altura, a altura total $(H)$ de todos os indivíduos com DAP $\geq 5 \mathrm{~cm}$. Todos os dados foram anotados em planilha de campo.

A análise florística foi realizada por meio da identificação de espécies, sendo que a análise da estrutura seguiu as recomendações de Brower et al. (1998), avaliada pela abundância ( $N=n_{i}$, onde: $n_{i}$ é o número de indivíduos da espécie), calculada em indivíduo $\left(\mathrm{n}_{\mathrm{i}}\right)$ por unidade de área (ha). Então, temos: indivíduo por hectare ( $\left.n_{i} / h a\right)$.

Para avaliar o EC contido na biomassa seca (BS) acima do solo, a vegetação dos SAF foi dividida em três componentes: açaí (Euterpe oleracea Mart.), cacau (Theobroma cacao L.) e árvores (espécies florestais). A BS foi determinada pelo método indireto, por meio das equações alométricas criadas por Bartelt et al. (2000): BS = exp $(-0,0550+0,0451 \times$ DAP $)$ e $B S=\exp (-0,0470+0,0750$ $\times$ DAP), respectivamente, $B S$ de folhas e de estipes de açaí; $\mathrm{BS}=\exp (-1,3200+0,0566 \times \mathrm{DAP})$ e BS $=\exp (0,0320$ $+0,0810 \times \mathrm{DAP})$, respectivamente, $\mathrm{BS}$ de folhas e do lenho de cacau e BS $=\exp (-0,7600+0,1242 \times$ DAP $)$ e BS $=$ $\exp (0,2040+0,3129 \times D A P)$, respectivamente, $B S$ de folhas e do lenho de árvores. $\bigcirc$ termo lenho engloba as partes aéreas das plantas: fuste, galhos e cascas.

A BS foi calculada em unidade de peso (t) por unidade de área (ha), para, depois, determinar o carbono (C) contido na BS (t C/ha). O EC em diversos estudos realizados em áreas naturais ou antrópicas tem sido obtido pela multiplicação da BS total (BS em cada parte da planta) por um fator igual a 0,45 , pois, segundo a literatura especializada, em média, ela contém cerca de $45 \%$ de carbono, variando entre 0,43 a 0,48 (Higuchi et al., 1998), sendo possível usar o fator 0,50, pois, conforme estudos do Intergovernmental Panel On Climate Change (Penman et al., 2003) e de Montagnini \& Nair (2004), esta seria a quantidade de carbono contida na biomassa vegetal. Neste estudo, optou-se pelo fator 0,45 , por ter sido observado em pesquisas realizadas com espécies nativas da Amazônia (Higuchi \& Carvalho Jr., 1994; Higuchi et al., 1998).

O modelo presa-predador de Lotka-Volterra é frequentemente usado para avaliar a interação entre populações de seres vivos (bióticas), por exemplo: tubarão $x$ peixes. Utilizá-lo para avaliar a interação entre componentes abiótico (carbono) e biótico (planta) foi, a princípio, um desafio. Entretanto, julgamos que a ciência e os métodos avançam assim. Havia, no entanto, algumas incertezas: o modelo produziria resultados coerentes e confiáveis? O carbono não tem eventos naturais de nascimento e de mortalidade. Assim, o 'nascimento' do carbono foi considerado pela produção natural ou por atividades antrópicas e a 'mortalidade', pela assimilação de carbono por plantas, via fotossíntese. A partir dessa conjectura, passamos a considerar o carbono como a 'população presa' e as plantas, a 'população predadora'.

Então, para a aplicação do modelo Lotka-Volterra, a 'presa' foi representada pelo EC e o 'predador' pelo número de plantas (açaí, cacau e árvores) encontradas nos SAF-CM e SAF-TA. A análise foi realizada no Matrix Laboratory (MATLAB), que é um software interativo 
direcionado para cálculos numéricos e gráficos científicos, seu ponto forte é a manipulação e o cálculo de autovalores e de autovetores, os cálculos e a fatoração de matrizes, a resolução de sistemas lineares e de equações diferenciais (Farina \& Posser, 2015). O modelo ficou assim representado:

$$
\begin{aligned}
& \text { PRESA } \\
& \text { (Carbono) } \\
& \times \\
& \begin{aligned}
& \frac{\partial \text { Carbono }}{\partial t}= \text { Emissão } \times \text { Carbono-Absorção } \times \\
& \text { Carbono } \times \text { Plantas }
\end{aligned} \\
& \begin{aligned}
& \frac{\partial \text { Plantas }}{\partial \mathrm{t}}=- \text { Plantio } \times \text { Plantas }+ \text { Desbaste } \times \\
& \text { Carbono } \times \text { Plantas }
\end{aligned}
\end{aligned}
$$

Onde:

a) taxa de crescimento da população presa $=$ emissão de carbono atmosférico;

b) taxa de decrescimento da população presa $=$ absorção de carbono atmosférico; c) taxa de crescimento da população predadora $=$ plantio nos SAF;

d) taxa de decrescimento da população predadora $=$ desbaste (corte, quedas etc.) nos SAF.

Este estudo não teve desígnio comparativo entre os SAF tradicional e comercial, nem buscou mostrar qual sistema seria o melhor ou o pior, mas sim a capacidade produtiva e a sua contribuição ambiental, através do EC e de interação das populações, demonstrada a partir da utilização do modelo presa-predador, configurado na apresentação dos eventos dos pontos de equilíbrio. $\mathrm{O}$ artigo, todavia, considerou algumas analogias e particularidades desses SAF, visando contribuir com a condução e o manejo desses importantes sistemas de uso a terra, orientando-os.

\section{RESULTADOS E DISCUSSÃO}

Nos SAF estudados em Cametá - SAF-CM 1, 2, 3 e 4 -, foi inventariado total de $9.832 \mathrm{n} / \mathrm{ha}$ (média de $2.458 \mathrm{n} / \mathrm{ha}$ ) e, nos de Tomé-Açu - SAF-TA 1, 2, 3 e 4 -, total de 4.996 $\mathrm{n}_{\mathrm{i}} / \mathrm{ha}$ (média de $1.249 \mathrm{n} / \mathrm{ha}$ ), com DAP $\geq 5 \mathrm{~cm}$ (Tabela 1).

\begin{tabular}{|c|c|c|c|c|c|c|c|c|c|c|c|c|}
\hline \multirow{3}{*}{-} & \multicolumn{12}{|c|}{$N\left(n_{i} / h a\right)$} \\
\hline & \multicolumn{6}{|c|}{ SAF-CM } & \multicolumn{6}{|c|}{ SAF-TA } \\
\hline & 1 & 2 & 3 & 4 & $M$ & e & 1 & 2 & 3 & 4 & $M$ & e \\
\hline Açaí & 1.872 & 1.484 & 1.352 & 504 & 1.303 & 15,97 & 171 & 95 & 543 & 520 & 332 & 12,75 \\
\hline Cacau & 68 & 496 & 984 & 1.380 & 732 & 21,13 & 1.044 & 472 & 1.123 & 651 & 823 & 10,87 \\
\hline Árvores & 572 & 408 & 284 & 428 & 423 & 5,74 & 101 & 89 & 105 & 83 & 95 & 1,05 \\
\hline Total & 2.512 & 2.388 & 2.620 & 2.312 & 2.458 & 42,84 & 1.316 & 656 & 1.771 & 1.253 & 1.249 & 24,67 \\
\hline \multirow{3}{*}{-} & \multicolumn{12}{|c|}{ EC (t/ha) } \\
\hline & \multicolumn{6}{|c|}{ SAF-CM } & \multicolumn{6}{|c|}{ SAF-TA } \\
\hline & 1 & 2 & 3 & 4 & $M$ & e & 1 & 2 & 3 & 4 & $M$ & e \\
\hline Açaí & 2,81 & 2,24 & 2,02 & 0,78 & 1,96 & 0,05 & 0,24 & 0,14 & 0,86 & 0,86 & 0,53 & 0,05 \\
\hline Cacau & 0,07 & 0,48 & 1,00 & 1,34 & 0,72 & 0,05 & 1,11 & 0,85 & 1,66 & 0,99 & 1,15 & 0,03 \\
\hline Árvores & 78,65 & 36,17 & 42,83 & 61,41 & 54,77 & 2,07 & 10,36 & 22,91 & 25,08 & 31,21 & 22,39 & 2,00 \\
\hline Total & 81,53 & 38,89 & 45,85 & 63,53 & 57,45 & 2,17 & 11,71 & 23,91 & 27,60 & 33,06 & 24,07 & 2,08 \\
\hline
\end{tabular}

Tabela 1. Número de plantas (N) e estoque de carbono (EC) encontrados em sistemas agroflorestais (SAF), estudados nos municípios de Cametá e de Tomé-Açu, no estado do Pará. As variáveis EC (t/ha) e N (n/ha) representam, respectivamente, a presa e o predador para análise do ponto de equilíbrio, utilizando-se o modelo presa-predador de Lotka-Volterra entre as populações desses SAF. Legendas: $M=$ médias; $e=$ erro padrão da média.

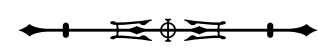


Nos SAF-CM, o açaí representou 53,0\% dos indivíduos, o cacau, 29,8\% e as árvores, 17,2\%; enquanto que, nos SAF-TA, o açaí representou $26,6 \%$ dos individuos, o cacau, 65,8\% e as árvores, 7,6\% (Figura 2).

O EC médio contido na BS acima do solo em relação à vegetação localizada nos SAF-CM foi de 1,86 t/ ha para o açaí $(3,4 \%)$, de 0,72 t/ha para o cacau (1,3\%) e de $54,77 \mathrm{t} /$ ha para as árvores (95,3\%), enquanto que, nos SAF-TA, foi de 0,53 t/ha para o açaí (2,2\%), de 1,15 t/ ha para o cacau (4,8\%) e de 22,39 t/ha para as árvores $(93,0 \%)$ (Tabela 1). O EC nos SAF-CM foi mais que o dobro em comparação com o encontrado nos SAF-TA. A diferença observada entre o EC dos SAF estudados pode estar relacionada ao manejo adotado nessas áreas e ao maior número de árvores, componente que aporta mais biomassa e, consequentemente, estoca mais carbono. Todavia, Murillo (1997) avalia que seria inadequado comparar qual tipo de sistema estocaria mais carbono, se o natural ou o plantado.

Ademais, seria natural considerarmos que os resultados decorram de agroecossistemas tradicionais originados de uma capoeira, por exemplo, como os SAFCM, por se tratar de povoamentos vegetais estabelecidos, maduros e, em alguns casos, em estado clímax, os quais, de certa forma, ainda mantêm parte da fisionomia, da estrutura e da fisiologia da vegetação original.

Para avaliar o ponto de equilíbrio entre as populações de presas e de predadores nos SAF-CM e SAF-TA, foram utilizados os resultados apresentados na Tabela 1 , empregados no processamento dos dados no modelo presa-predador no software MATLAB.

Nos SAF-CM, no primeiro ano de manejo, 1991 (SAF-1), 1990 (SAF-2), 1988 (SAF-3) e 1987 (SAF-4), a população de plantas (predador) era menor em relação à de carbono (presa), mas é notável a evolução na taxa de crescimento a partir do ano seguinte: 1992 (SAF-1), 1991 (SAF-2), 1989 (SAF-3) e 1988 (SAF-4), sendo maior na população predadora, com destaque para os SAF 1 e 4 , começando a ocorrer taxa de decrescimento (redução)

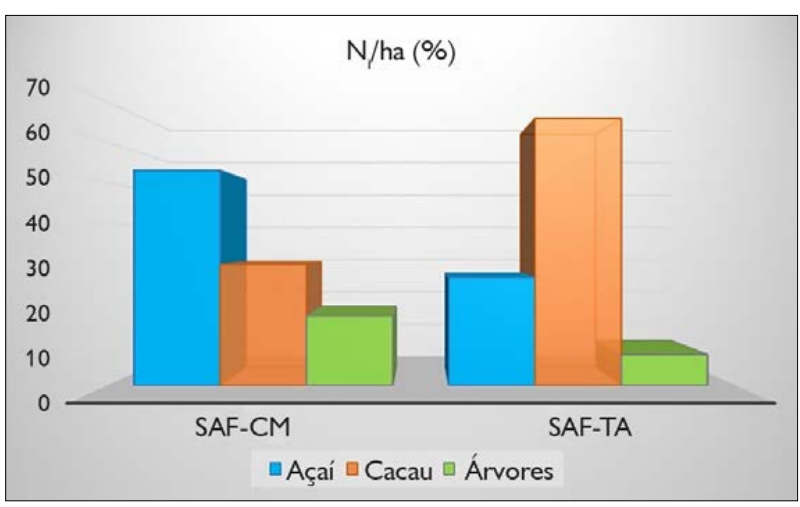

Figura 2. Abundância ( $\mathrm{n}$ /ha) de espécies encontradas em sistemas agroflorestais (SAF) estudados nos municípios de Cametá (SAF-CM) e de Tomé-Açu (SAF-TA), no estado do Pará.

na presa. As duas populações chegam ao equilíbrio: SAF-1 em meados do ano de 1991, SAF-2 em meados de 1990, SAF-3 no final de 1988 e SAF-4 no final de 1987. Um novo ponto de equilíbrio foi observado no SAF-1 em meados dos anos 2000, no SAF-2 em meados de 1997, no SAF-3 em meados de 1995 e no SAF-4 no final de 1995 (Figura 3).

Como o modelo presa-predador é um sistema que apresenta oscilações, ora aumenta ora diminui, existem períodos ou intervalos de tempo nos eventos de equilíbrio: no SAF-1 em 1991 e 2000, no SAF-2 em 1990 e 1997, no SAF-3 em 1988 e 1995 e no SAF-4 em 1987 e 1995, havendo projeção gráfica de novos eventos entre 2001-2010 (SAF-1), 1997-2004 (SAF-2), 1995-2002 (SAF3) e 1995-2003 (SAF-4). A defasagem na evolução das populações foi de cerca de seis meses nos SAF 1, 3 e 4 e de um ano no SAF-2, com média de 7,5 meses (Figura 3).

Analisando-se a evolução das populações de presa e de predador, nota-se que chegaram ao ponto máximo (ápice), respectivamente, com valores absolutos: no SAF-1, de 110.000 e 135.000 e equilíbrio em 75.000; no SAF-2, de 70.000 e 90.000 e equilíbrio em 50.000; no SAF-3, de 75.000 e 95.000 e equilíbrio em 53.000; e no SAF-4, de 95.000 e de 125.000 e equilíbrio em 65.000, sendo sempre maior a evolução da população predadora (Figura 3). 


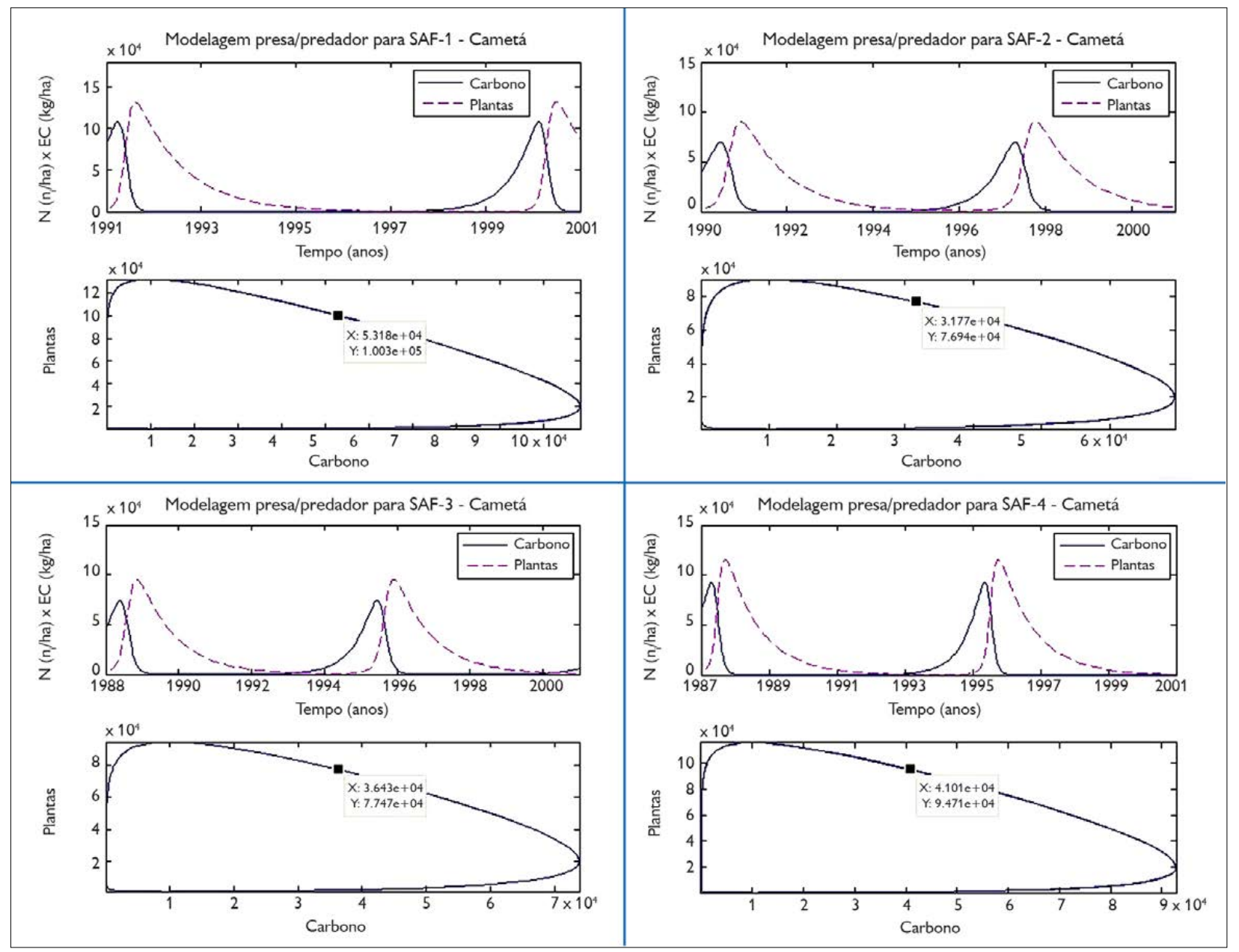

Figura 3. Modelagem da interação e do ponto de equilíbrio na assimilação de carbono em sistemas agroflorestais (SAF) estudados no município de Cametá, Pará, utilizando as equações diferenciais simultâneas (EDS), modelo presa-predador de Lotka-Volterra.

Na parte inferior do gráfico, uma elipse (trajetória) mostra a relação numérica entre as populações (concentração) nos SAF-CM, onde o ponto de equilíbrio foi obtido nos intervalos já evidenciados, nos respectivos valores absolutos: no SAF-1, foi de 53.180 kg C e 100.300 $n_{i}$, em 1996; no SAF-2, de 31.770 kg C e $76.940 n_{i}$, em 1995; no SAF-3, de 36.430 kg C e 77.470 n, em 1994; e no SAF-4, de 41.010 kg C e 94.710 n, em 1993 (Figura 3).

A assimilação de carbono foi maior no período de 1991-1993 (maior evento) e de 2000-2001 no SAF-1, de 1991-1992 e 1997-1998 no SAF-2, de 1989-1990 e 19961997 no SAF-3 e de 1998-1999 e 1996-1997 no SAF-4.
O ano de 1997 foi mais propício à assimilação de carbono nos SAF-CM e no biênio 1996-1997, com registro em dois SAF (Figura 3).

Nos SAF-TA, no primeiro ano de manejo, 2012 (SAF1), 2006 (SAF-2), 2000 (SAF-3) e 1994 (SAF-4), a população de plantas (predador) era menor em relação à de carbono (presa), mas nota-se evolução na taxa de crescimento a partir do ano seguinte: 2013 (SAF-1), 2007 (SAF-2), 2001 (SAF-3) e 1995 (SAF-4), sendo maior na população predadora, com destaque para os SAF 2 e 4, começando a ocorrer taxa de decrescimento (redução) na presa, com pequeno acréscimo no SAF-3 em meados de 2014. As duas populações chegam 
ao equilíbrio: SAF-1 no início do ano de 2013, SAF-2 no início de 2007, SAF-3 no início de 2001 e SAF-4 no início de 1995. O SAF-1 só apresentou um evento de equilíbiro, devido à pouca idade (três anos). Porém, nos demais SAF, novo ponto de equilíbrio foi observado no SAF-2 em meados do ano de 2014, no SAF-3 em meados de 2007 e no SAF-4 no início de 2002. Além disso, outro ponto de equilíbrio foi observado nos SAF mais velhos (15 e 21 anos): no SAF-3 no início do ano de 2014 e no SAF-4 em meados de 2009 (Figura 4).

O modelo presa-predador é oscilante, dessa forma apresenta variações observadas nos intervalos de tempo e nos eventos de equilíbrio: no SAF-1 em 2013, no SAF-2 em 2007 e 2014, no SAF-3 em 2001, 2007 e 2014 e no
SAF-4 em 1995, 2002 e 2009, havendo projeção gráfica de novos eventos entre 2015-2018 (SAF-1), 2014-2021 (SAF 2 e 3) e 2009-2016 (SAF-4). A defasagem na evolução das populações foi de cerca de seis meses no SAF-1 e de um ano nos SAF 2, 3 e 4, com média de 10,5 meses (Figura 4).

$\mathrm{Na}$ análise do ápice da evolução das populações de presa e de predador, observou-se, respectivamente: no SAF-1, de 60.000 e 85.000 e equilíbrio em 51.000; no SAF-2, de 90.000 e 115.000 e equilíbrio em 65.000; no SAF-3, de 70.000 e 95.000 e equilíbrio em 48.000; e no SAF-4, de 80.000 e de 100.000 e equilíbrio em 63.000, sendo sempre maior a evolução da população de plantas (Figura 4).

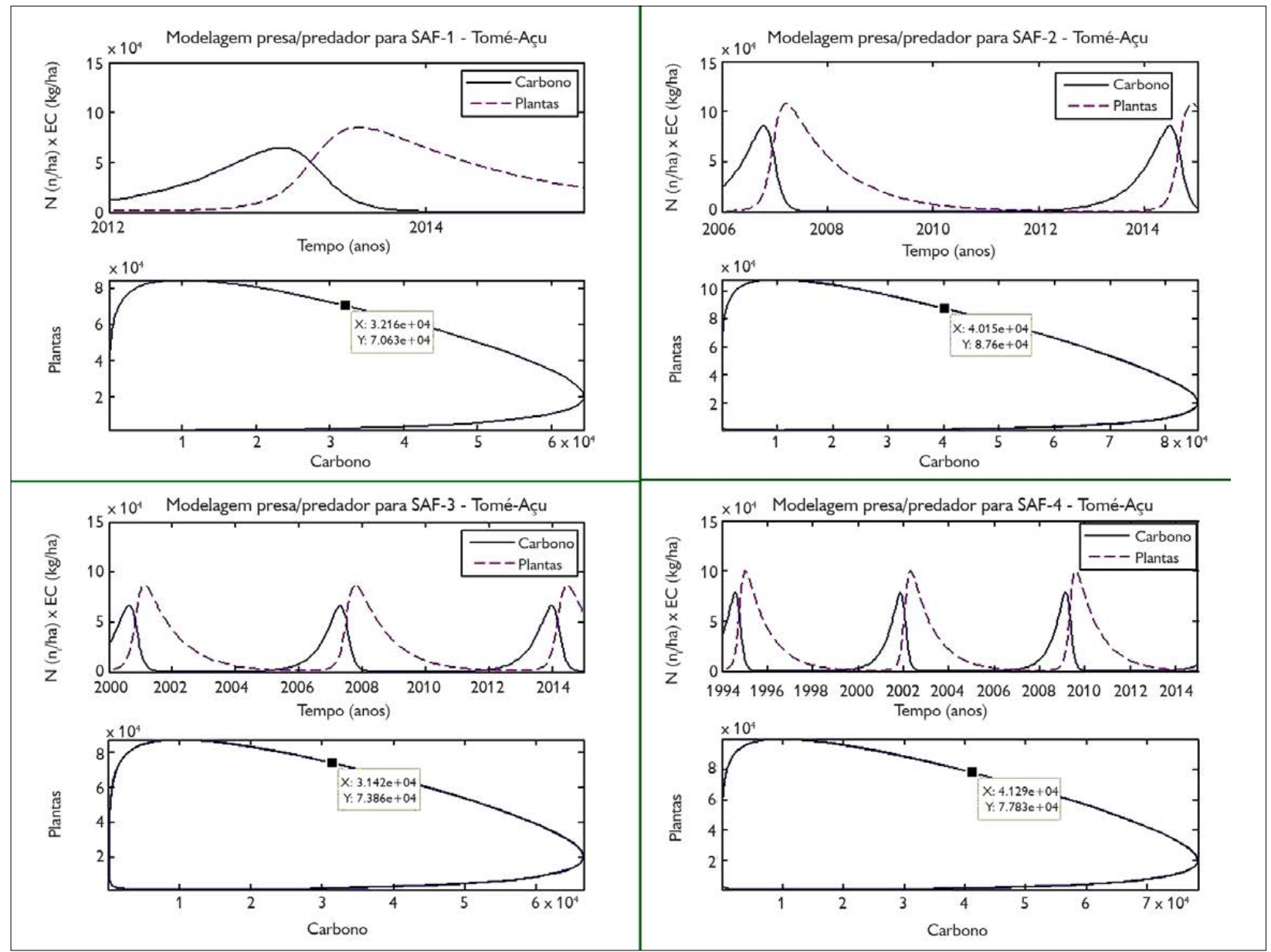

Figura 4. Modelagem da interação e do ponto de equilíbrio na assimilação de carbono em sistemas agroflorestais (SAF) estudados no município de Tomé-Açu, Pará, utilizando as equações diferenciais simultâneas (EDS), modelo presa-predador de Lotka-Volterra.

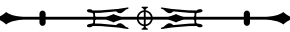


A trajetória mostra graficamente a relação numérica entre as populações de presa e de predador nos SAF-TA, onde o ponto de equilíbrio foi obtido nos intervalos de tempo: no SAF-1, foi de $32.160 \mathrm{~kg}$ C e $70.630 \mathrm{n}_{\mathrm{i}}$, em 2013; no SAF-2, de $40.100 \mathrm{~kg} C$ e 87.600 n; , em 2010; no SAF3, de $31.420 \mathrm{~kg}$ C e $73.860 \mathrm{n}_{i}$, em 2007; e no SAF-4, de $41.290 \mathrm{~kg} \mathrm{C} \mathrm{e} 77.830$ n, em 2005 (Figura 4).

A assimilação de carbono foi maior no período entre os anos de 2013-2014 no SAF-1, entre 2007-2009 e 2014-2015 no SAF-2, entre 2001-2002, 2007-2009 (maior evento) e 2014-2015 no SAF-3 e entre 1994-1996, 2002-2003 e 2009-2011 no SAF-4. O ano de 2014 foi mais propício à assimilação de carbono nos SAF-TA, com registro em três SAF, e no biênio 2014-2015, com registro em dois SAF (Figura 3).

Neste estudo, observou-se a sucessão oscilante na evolução das populações de presas e de predador, onde sempre a primeira (presa) é maior no início, mas é alcançada e superada pela segunda (predadora). O que é positivo, exitoso e desejado, do ponto de vista ambiental e socioeconômico. Então, poderíamos dizer que os SAF estudados estariam cumprindo alguns de seus atributos, como contribuir para minorar os agravos ambientais causados por atividades antrópicas, reduzindo a concentração de $\mathrm{CO}_{2}$ atmosférico, principal causador do efeito estufa.

Como a interação se dá entre uma população biótica (plantas) e outra abiótica (carbono), ecologicamente, o ideal seria que, a partir do ponto de equilíbrio, não tivessem mais oscilações entre as populações, mas somente a evolução da população de plantas (predadora), o que significaria maior eficiência na assimilação de carbono, gerando, em tese, um superávit no 'sequestro de carbono'. Todavia, em razão da influência mútua entre as populações vivas ou bióticas, é imprescindível que se tenha ou se mantenha o equilíbrio nas relações e nas interações entre as espécies, para o perfeito funcionamento dos ecossistemas naturais.

Outro fato a ser considerado seria verificar qual a defasagem de tempo na evolução dessas populações (carbono e plantas) e sua real oscilação, estabelecendo valores ou intervalos no tempo, visando identificar ou mesmo presumir um padrão de interação, haja vista que, quando se trata de interação entre espécies animais - uma presa e outra predadora -, o ideal seria haver oscilação entre essas populações - ora elas crescem, ora elas reduzem -, em uma interação em que uma regula a quantidade e/ou a existência da outra. A repetição no padrão dos gráficos representa o equilíbrio entre as populações presa e predadora. Esse processo ocorre em um ciclo que se repete e se renova no tempo, o que é importante e salutar à seleção natural nos sistemas biológicos, onde prevalecem e sobrevivem os indivíduos mais fortes e as espécies mais adaptadas, em consenso com a teoria da evolução das espécies, de Charlie Darwin (1861).

A revisão da literatura sobre modelagem matemática de sistemas e de ecologia numérica evidenciou que o modelo presa-predador de Lotka-Volterra nunca havia sido utilizado para analisar a dinâmica e a interação entre uma população abiótica (carbono) e uma população biótica (plantas). O único e mais similar estudo foi o feito por Aviz dos Santos (2011) entre populações de árvores (presa) e humana (predador), ambas bióticas. Entretanto, esta autora, na busca por referências na literatura, também se deparou com uma situação semelhante, haja vista, segundo pesquisadores consultados, não haver nenhum nível trófico direto entre humanos e árvores, sugerindo que o modelo presa-predador, nesse caso, fosse adaptado e chamado de recurso-consumidor, sendo o recurso equivalente às árvores e o consumidor aos homens, o que foi acatado naquela pesquisa por se tratar de duas populações bióticas.

A TGS seria um instrumento útil e capaz de fornecer modelos a serem utilizados em diferentes campos do conhecimento e transmitidos de uns para os outros, salvaguardando-os do risco de analogias ilusórias. A reciprocidade tratada por essa teoria é muito mais do que uma analogia, na medida em que possibilita aplicação de abstrações correspondentes e de modelos conceituais a fenômenos de diferentes naturezas, residindo nesse todo o valor da teoria (Bertalanffy, 1968). Por isso, e pelo fato 
de o modelo ser oscilatório, é notável que, na evolução dos gráficos, neste estudo, por várias vezes as 'linhas' de plantas e de carbono chegaram a zero na quantidade de suas populações, o que não configura a verdade no ambiente e nos SAF, apontando para o modelo, que, na realidade, deve ser visto e analisado de maneira qualitativa (Chase et al., 2014). Com aumento da população de plantas, a população de carbono diminui, como consequência das interações entre presa-predador. Supõe-se que este efeito seja proporcional ao produto de ambas as populações (- absorção x carbono $x$ plantas), enquanto a população de predadores aumenta ( + desbate $\times$ carbono $\times$ plantas), de forma semelhante.

\section{CONCLUSÕES}

Nos SAF estudados em Cametá (SAF-CM) e em ToméAçu (SAF-TA), o açaí e o cacau foram as espécies mais importantes. Entre as árvores, destacaram-se: Virola surinamensis (Rol. ex Rottb.) Warb., Hevea brasiliensis (Willd. exA. Juss.) Müll. Arg. e Carapa guianensis Aubl., nos SAF-CM, e Spondias lutea L., Bertholletia excelsa Bonpl. e Swietenia macrophylla King, nos SAF-TA.

Os SAF estudados demonstraram capacidade de prestar relevantes serviços ambientais e geração de renda, se houver remuneração nesses serviços, além de contribuírem tanto na assimilação de carbono e na mitigação da ação danosa dos GEE na atmosfera quanto na redução de áreas desmatadas e do avanço da fronteira agrícola, minorando os agravos ambientais e socioeconômicos e, ainda, ou melhor, propiciando a manutenção da biodiversidade.

O emprego do modelo presa-predador na análise da interação entre as populações de plantas e de carbono demonstrou que os SAF estudados apresentaram elevado número de ponto de equilíbrio (populações proporcionais ou estáveis). Na prática, este seria o melhormomento para conduzir um plano de manejo que vise ambiência e remuneração de serviços ambientais, como o crédito de carbono.

O modelo presa-predador mostrou-se suscetível e eficiente na avaliação do ponto de equilíbrio entre as populações de carbono (abiótica) e de plantas (biótica) cultivadas em SAF, ou seja, não é somente voltado à espécie comercial, mas também à cobertura vegetal compondo o sistema e às suas interações internas e externas, com o ambiente do seu entorno (a vegetação, o solo, a água). Tudo isso ratifica os SAF como uma alternativa de produção sustentável do ponto de vista ambiental e socioeconômico para os municípios de Cametá e de Tomé-Açu, bem como para as regiões adjacentes e para a Amazônia.

\section{AGRADECIMENTOS}

Agradeço a Deus por tudo e aos proprietários das áreas estudadas em Cametá - Sr. Bráulio Leão, Sr. Raimundo Lousada, Sr. Wilson Pinto e Sra. Maria Amélia, e suas famílias - e em Tomé-Açu - Sr. Michinori Konagano e Sr. José Maria Mendes, e suas famílias.

\section{REFERÊNCIAS}

AVIZ DOS SANTOS, M. A. B., 2011. Ciências Naturais e Ciências Sociais: conceitos e operadores transversais na perspectiva sistêmica: o caso da interação: 1-100. Tese (Doutorado em Ciências Agrárias) - Universidade Federal Rural da Amazônia, Belém.

BARROS, A. V. L., 2009. Evolução dos sistemas agroflorestais desenvolvidos pelos agricultores nipo-brasileiros no município de Tomé-Açú, Pará: 1-100. Tese (Doutorado em Ciências Agrárias) - Universidade Federal Rural da Amazônia, Belém.

BARTELT, D., J. KOCH \& M. M. TOURINHO, 2000. Anbau von Acai (Euterpe oleracea) und Kakao (Theobroma sylvestre) in Primärwäldern der varzeas am rio Tocantins (Brasilien/Para). Forstarchiv 71(6): 250-256.

BERTALANFFY, L., 1968. General system theory. Foundations, development and applications: 1-277. George Braziler, New York.

BERTALANFFY, L., 1975. Perspectyvas en la teoría general de sistemas. Estudios científico-filosóficos: 1-283. Alianza Editorial, Madrid.

BROWER, J. E., J. H. ZAR \& C. N. ENDE, 1998. Field and laboratory methods for general ecology: 1-273. McGraw, New York.

CHASE, O. A., J. F. S. ALMEIDA, J. R. B. SOUZA \& C. T. C. JUNIOR, 2014. Sensory platform architecture for IN SITU monitoring the thermal comfort in rural environments - the case study at Federal Rural University of Amazonian, Brazil. Measurement 58: 294-300. DOI: https://doi.org/10.1016/j.measurement.2014.08.031. 
DALLAGNOL, F. S., F. MOGNON, C. R. SANQUETTA \& A. P. D. CORTE, 2011. Teores de carbono de cinco espécies florestais e seus compartimentos. Floresta e Ambiente 18(4): 410-416. DOI: http:// dx.doi.org/10.4322/floram.2011.060.

DARWIN, C., 1861. On the origin of species by means of natural selection, or the preservation of favoured races in the struggle for life. D. Appleton and Company, New York.

DIAS, I. P., B. C. COSTA, M. M. TOURINHO \& J. F. ALMEIDA, 2015. Avaliação da estimativa de fixação de carbono em sistemas agroflorestais na região amazônica. Revista Verde 10(5): 7-10. DOI: https://doi.org/10.18378/rvads.v10i5.3411.

FARINA, L. A. \& M. S. POSSER, 2015. Matrix Laboratory- MATLAB Ferramenta matemática para Engenharia: 1-31. Universidade Federal do Rio Grande do Sul, Porto Alegre.

FASSBENDER, H. W., 1993. Modelos edafológicos de los sistemas de produción agroforestales: 2. ed.: 1-29. Centro Agronomico Tropical de Investigacion y Enseñanza, Turrialba.

HAIRIAH, K., S. M. SITOMPULL, M. NOORDWIJK \& C. PALM, 2001. Methods for sampling carbon stocks above and below ground. In: M. NOORDWIJK, S. WILLIAMS \& B. VERBIST (Ed.): Towards integrated natural resource management in forest margins of the humid tropics: local action and global concerns: 1-49. ICRAF, Bogoi.

HIGUCHI, N. \& A. J. CARVALHO JR., 1994. Fitomassa e conteúdo de carbono de espécies arbóreas da Amazônia. Anais do Seminário Emissão e Sequestro de $\mathrm{CO}_{2}$ - uma nova oportunidade de negócios para o Brasil 125-153.

HIGUCHI, N., J. SANTOS, R. J. RIBEIRO, L. MINETTE \& Y. BIOT, 1998. Biomassa da parte aérea da vegetação de floresta tropical úmida de terra-firme da Amazônia brasileira. Acta Amazonica 28(2): 153-165. DOI: http://dx.doi.org/10.1590/1809-43921998282166.

HOMMA, A. K. O., 2006. Organização da produção e comercialização de produtos agropecuários: o caso da colônia agrícola nipo-brasileira de Tomé-Açu, Pará. In: L. J. M. VILCAHUAMÁN, J. RIBASKI \& A. M. B. MACHADO (Ed.): Sistemas agroflorestais e desenvolvimento com proteção ambiental: perspectivas, análise e tendências: 51-77. EMBRAPA Florestas, Colombo.

INSTITUTO BRASILEIRO DE GEOGRAFIA E ESTATÍSTICA (IBGE), 2012. Censo demográfico 2010: 1-211. IBGE, Rio de Janeiro.

KETTERINGS, Q. M., R. COE, M. NOORDWIJK, Y. AMBAGAU \& C. A. PALM, 2001. Reducing uncertainty in the use of allometric biomass equations for predicting above-ground tree biomass in mixed secondary forests. Forest Ecology and Management 146(13): 199-209. DOI: https://doi.org/10.1016/S0378-1127(00)00460-6.

LÜTZ, A. F., 2011. Competição e coexistência em populações biológicas: 1-38. Monografia (Bacharelado em Física) - Universidade Federal do Rio Grande do Sul, Porto Alegre.
MONTAGNINI, F. \& P. K. R. NAIR, 2004. Carbon sequestration: an underexploited environmental benefit of agroforestry systems. Agroforestry Systems 61: 281-295. DOI: http://dx.doi.org/10.1023/ B:AGFO.0000029005.92691.79.

MURILLO, M. A., 1997. Almacenamiento y fijación de Carbono en ecosistemas forestales. Revista Forestal Centroamericana 6(19): 9-12.

ODUM, H. T., E. C. ODUM, M. T. BROWN, D. LAHART, C. BERSOK, J. SENDZIMIR, B. S. GRAEME, D. SCIENCEMAN \& N. MEITH, 1987. Environmental systems and public policy. Ecological Economics Program. University of Florida, Gainesville.

OSTERROOHT, von M., 2002. Manejo de Sistemas Agroflorestais - SAF's. Agroecologia 15: 12-13.

PENA-VEGA, A., 2003. O despertar ecológico: Edgar Morin e a ecologia complexa: 1-109. Garamond, Rio de Janeiro.

PENMAN, J., M. GYTARSKY, T. HIRAISHI, T. KRUG, D. KRUGER, R. PIPATTI, L. BUENDIA, K. MIWA, T. NGARA, K. TANABE \& F. WAGNER, 2003. Good practice guidance for land-use change and forestry: 1-593. IPCC, Japan.

PORRO, R., R. P. MILLER, M. R. TITO, J. A. DONOVAN, J. L. VIVAN, R. TRANCOSO, R. F. VAN KANTEN, J. E. GRIJALVA, B. L. RAMIREZ \& A. L. GONÇALVES, 2012. Agroforestry in the Amazon region: a pathway for balancing conservation and development. In: P. K. R. NAIR \& D. GARRITY (Ed.): Agroforestry: the future of global land use: 391-428. Springer (Advances in Agroforestry, 9), Dordrecht.

SANTOS, S. R. M., I. S. MIRANDA \& M. M. TOURINHO, 2004 Estimativa de biomassa de sistemas agroflorestais das várzeas do rio Juba, Cametá, Pará. Acta Amazonica 34(1): 1-8. DOI: http://dx.doi. org/10.1590/S0044-59672004000100001.

SANTOS, S. R. M., O. R. KATO \& M. M. TOURINHO, 2019. Diversidade florística e estoque de carbono de sistemas agroflorestais em dois municípios do nordeste paraense, Brasil. Boletim do Museu Paraense Emílio Goeldi. Ciências Naturais 14(1): 31-42.

SMITH, N., J. DUBOIS, D. CURRENT, E. LUTZ \& C. CLEMENT, 1998. Agroforestry experiences in the Brazilian Amazon: constraints and opportunities: 1-146. Pilot Programme to Conserve the Brazilian Rain Forest/World Bank, Brasília.

SOUGATA, B. \&J. SHIBU, 2012. Agroforestry for biomass production and carbon sequestration: an overview. Agroforesty Systems 86(2): 105-111. DOI: https://doi.org/10.1007/s10457-012-9573-x.

VILLA, P. M., S. V. MARTINS, L. MONSANTO, S. N. OLIVEIRANETO \& N. M. CANCIO, 2015. La agroforestería como estrategia para la recuperación y conservación de reservas de carbono en bosques de la Amazonía. Bosque 36(3): 347-356. DOI: http:// dx.doi.org/10.4067/S0717-92002015000300002. 